\title{
ETINOBIOLOGY AND CONSERVAULON
}

\section{The role of individuals in the resilience of local medical systems based on the use of medicinal plants - a hypothesis}

\author{
Washington Soares Ferreira Júnior ${ }^{1}$, Flávia Rosa Santoro ${ }^{1}$, André Luiz \\ Borba Nascimento ${ }^{1}$, Ana Haydée Ladio ${ }^{2}$, Ulysses Paulino Albuquerque ${ }^{1_{*}}$ \\ ${ }^{1}$ Laboratório de Etnobotânica Aplicada, Departamento de Biologia, Universidade Federal Rural de \\ Pernambuco, Av. Dom Manoel de Medeiros, s/n, Dois Irmãos, CEP: 52171-900, Recife, PE, Brasil \\ ${ }^{2}$ Universidad Nacional del Comahue, Quintral, 1250-8400 San Carlos de Bariloche, Río Negro, \\ Argentina \\ * Corresponding author \\ $\triangle$ E-mail address: UPA (upa@db.ufrpe.br)
}

Ethnobiology and Conservation, 2:1 (14 May 2013)

ISSN 2238-4782

doi: $10.15451 /$ ec 2013-8-2.1-1-10

ethnobioconservation.com

\section{Abstract}

Some studies in the field of ethnobiology investigate the knowledge and use of biological resources, especially medicinal plants, to infer the resilience of local medical systems. However, little is known about the resilience of such systems in the context of the distribution of knowledge among individuals in the studied communities. For example, although a medical system is formed by a diverse set of species, the distribution of knowledge may be limited to a small number of individuals, which reduces its flexibility and does not favor its resilience. Thus, this manuscript proposes the hypothesis that the medical system appears resilient when assessing the knowledge of the individuals who compose the system. The knowledge of medicinal plants for the treatment of therapeutic targets among the individuals is used as an example. Additionally, predictions are made and a methodological protocol is described to test the hypothesis by a simple analysis.

Keywords: Medical ethnobiology - resilience - individual traditional knowledge therapeutic targets 


\section{Background}

Various human groups in distinct environments are related to conditions that alter their health status, leading the groups to form medical systems. In this manuscript, we understand a medical system to be a cultural system, as defined by Kleinman (1978), made up of knowledge shared by a human group related to the identification of illnesses, locally known associated symptoms and causes, and strategies developed to resolve such health issues.

Regarding the treatment of illnesses, several studies have contributed to the knowledge of how plant resources are incorporated in medical systems as a culturally shared strategy for the treatment and/or prevention of illnesses (Ankli et al. 1999; Stepp \& Moerman 2001; Waldstein \& Adams 2006; Alencar et al. 2010). Considering that medical systems feature a strong adaptive component, some researchers seek to investigate the resilience of such systems by assessing features of the system that lead to the maintenance of plant use and thereby the health of the persons who participate in the system (Ladio \& Lozada 2004, 2008, 2009; Albuquerque \& Oliveira 2007; Ferreira Júnior et al. 2011). For example, Albuquerque \& Oliveira (2007) argue that categories of illnesses with few options of species for their treatment create more vulnerability than local categories of illnesses with several options of species, based on the assumption that any disturbance that reduces the availability of medicinally useful species at any given moment can seriously affect the categories with few available species. Such categories with few species reduce the flexibility of the system in the face of "disturbances", suggesting points of low resilience (see Ladio \& Lozada 2004).

Resilience is defined in this work as the capacity of a system to absorb disturbances and reorganize after their occurrence to maintain the system's functions (Holling 1973). It is important to consider resilience as a multi-dimensional property that involves various factors acting together. Therefore, resilience is very difficult to measure directly, even if all the variables are considered individually. However, an acceptable assumption to allow an approximation of the phenomenon requires that the system under study exhibit an expected behavior, functioning within the normal limits of a natural system (Ungar 2012).

In the ethnobiological literature, when considering the studies of the resilience of medical systems, the functional perspective of those systems has still barely been explored. Current studies of resilience have explored the use and knowledge of resources by groups of people but do not consider the distribution of that use/knowledge among individuals and its implications for the resilience of the local medical system. The study of the distribution of knowledge allows patterns of consensus and variations of knowledge to be understood in human populations (see Hopkins \& Stepp 2012). In addition, such a study enables the factors that lead to this variation to be understood. Many studies have found that knowledge about medicinal plants is not distributed evenly, with differences in knowledge according to socioeconomic factors (Hanazaki et al. 2000; Quinlan \& Quinlan 2007; Silva et al. 2011) and depending on the mechanisms of cultural transmission (see Cavalli-Sforza \& Feldman 1981).

In this sense, the distribution of the knowledge of individuals in a medical system is influenced by many factors. However, even with this complexity, the study of this distribution can provide important information about the resilience of local systems. For example, although a medicinal category has many options of species 
for treatment in a system, that category may be known only by few local inhabitants, which would lead to greater vulnerability of that category compared to others that are well-known by most people (see Ferreira Júnior et al. 2011). Similarly, a system may involve many illnesses with various options of species, thus indicating various points of resilience; however, most people know few plants to treat those illnesses, leading to greater vulnerability of those people. This example demonstrates that the distribution of knowledge in the population has a key role in understanding resilience. If that knowledge is not shared and perpetuated (by cultural transmission) the system does not function flexibly.

This paper presents a first attempt to consider the distribution of knowledge to sum up the interpretations regarding the resilience of local medical systems. The present manuscript proposes a hypothesis and a study design that considers the knowledge among the individuals in a community or population as a unit of analysis, to assess the contribution of the actors in a medical system to the resilience of that system. Thus, we present the following research question: is the local medical system resilient based on assessments of individual knowledge?

\section{Introducing the hypothesis}

In accordance with the background above, this manuscript proposes the hypothesis that a local medical system proves resilient when considering individual knowledge of medicinal plants (Table 1). This hypothesis assumes that in a medical system based on the use of plants, an individual who knows many therapeutic targets treated with various medicinal plants may be considered less vulnerable to adversities, in the sense that he/she can better maintain his/her health by taking advantage of resources available in the environment (see Ladio \& Lozada 2008; Ungar 2011) compared to other individuals who know few illnesses and few plants. The performance of this practice by many individuals allows the system to remain functional over time by cultural transmission, making it possible to observe the role of individuals in the resilience of the system. Therefore, it is expected that for a local medical system in which there is no strong unbalancing factor, the distribution of knowledge among individuals takes the shape of a normal curve. To test the present hypothesis, it is necessary to describe some assumptions on which our idea was constructed.

The hypothesis proposed herein is based on an ideal scenario, in which there are not strong unbalancing factors that generate discrepancies in the distribution of knowledge within a local medical system. For example, an individual's age and gender are assumed to be variables that do not strongly influence the distribution of local knowledge. Medical systems in which these factors have a strong influence can cause the distribution of knowledge to depart from the shape of a normal curve. Furthermore, mechanisms of cultural transmission should also be considered as generators of variation in the distribution. Cavalli-Sforza \& Feldman (1981) proposed models of knowledge transmission that incorporate vertical transmission, which occurs through contact between members of different generations of the same family, and horizontal transmission, which occurs through contact between members of different families. The authors reported that a more heterogeneous structure of knowledge within a system can be expected in the mechanisms of vertical transmission, as individuals do not communicate new knowledge (or innovations) with each other over time. However, a system characterized by horizontal 
transmission produces less heterogeneous knowledge between individuals. Accordingly, the proposed hypothesis proposed suggests that the local system contains mainly horizontal mechanisms of transmission, minimizing discrepancies among individuals.

Based on this ideal situation, most individuals are expected to share a mean amount of knowledge with each other, and few individuals are expected to share much and little knowledge, i.e., a distribution of knowledge that fits a normal curve. By applying this prediction to understand the resilience of medical systems, we present the following theoretical scenarios under which our hypothesis can be tested.

\section{Scenario 1: illness as a disturbance of the medical system}

Studies in medical anthropology have discussed how diseases have influenced human evolution over time (see Brown 1987; Wiley 1992). Some authors claim that the formation of medical systems can be considered an adaptive response to the resolution of health problems (Dunn 1976; Kleinman 1978; Henrich \& McElreath 2003). Thus, we assume that diseases are disturbances that can lead to greater changes in medical systems, and consequently, individuals of a cultural group who know the treatment for many illnesses through the use of plants can be considered less vulnerable than those who can only treat a few illnesses by using plants, regardless of the number of plant species known for each illness.

Thus, the following prediction can be developed to accept the proposed hypothesis, considering illness as the level of disturbance of a local medical system: It is expected that the knowledge of individuals of a given local population regarding treatment with medicinal plants follows a normal distribution, which would show that most individuals report nearly half of the illnesses known by local experts, or those people who exhibit the highest levels of knowledge in the community. This situation may lead to less vulnerability for most of the individuals studied in the case of illness and promote the resilience of the system. A different situation would be a non-normal data distribution, with many discrepancies, exhibiting both many individuals reporting many illnesses treated with plants (less vulnerable) and a high proportion of individuals reporting few illnesses that can be treated with plants (more vulnerable) (Figure 1).

Scenario 2: disturbances that can threaten the availability of medicinal species in a medical system

Other disruptions can threaten local medical systems, in the sense that such disruptions can alter the availability of medicinal species. Disruptions may include local practices, deforestation, climate changes, and other factors. The notion of utilitarian redundancy, proposed by Albuquerque \& Oliveira (2007), can be interesting for observing aspects of resilience of local systems facing such disturbances (see also Ladio \& Lozada 2004). One redundant medicinal category is that which features many species for treatment; this flexibility does not occur in low-redundancy or nonredundant categories in which there are few species or only one for treatment, respectively. When considering the dynamics of medical systems, redundancy has a functional interpretation for the system, in the sense that a large number of species in a medicinal category can provide greater flexibility during selection when one species is no longer available in the environment (Albuquerque \& Oliveira 2007). 
In this scenario, an individual who knows many species to treat his/her illnesses is less vulnerable than one who knows few species. A second prediction can be constructed to accept the proposed hypothesis, considering the disturbances that threaten the availability of medicinal species: It is expected that most of the individuals in a studied community will be concentrated around the mean for the number of species reported for each illness, or redundancy, a situation that once again takes the shape of a normal distribution (see Figure 1).
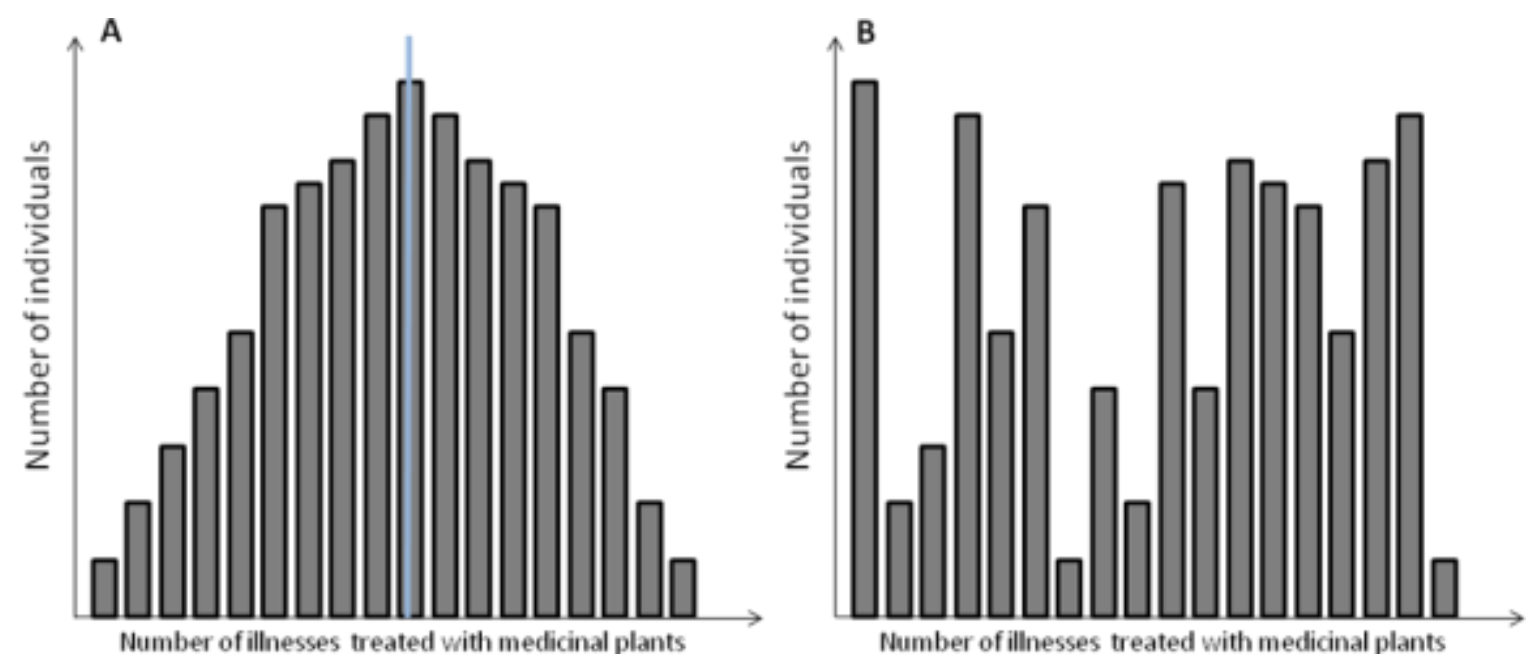

Figure 1. Possible results for the distribution of respondents regarding the number of illnesses reported as treatable with medicinal plants. (A) The expected situation for the confirmation of the hypothesis, in which the distribution of knowledge among individuals add to the resilience of the system. In the situation shown, it is expected that a greater proportion of individuals will be around the mean (indicated in blue on the graph) for the number of reports by each respondent. (B) The situation in which the hypothesis is rejected, in which a large proportion of individuals report many illnesses but another large proportion also reports few illnesses treatable with medicinal plants. These two situations may also be possible results for a distribution of respondents regarding the number of species used for each of the illnesses reported. 
Table 1. Hypothesis and predictions to assess the resilience of a medical system based on the knowledge of each individual that composes it. Scenario 1 considers illness as a disturbance of a local medical system; Scenario 2 considers disturbances that lead to a decrease in the availability of medicinal species in local medical systems.

Hypothesis

Scenario 1 Prediction

Scenario 2 Prediction
A local medical system proves resilient when it displays the expected behavior, functioning within the normal limits of a natural system

The distribution of knowledge among individuals based on the number of illnesses reported is expected to follow a normal distribution from a probabilistic perspective.

The distribution of individuals based on the number of plants reported for treating each therapeutic target is expected to follow a normal distribution from a probabilistic perspective.

\section{Testing the hypothesis}

We offer a simple methodological protocol to test the proposed hypothesis. When working with a local community, for example, it is important to first identify the elements of their medical system, such as (1) the set of therapeutic targets that are treated with local medicinal plants and (2) the set of plants reported for treatment of therapeutic targets. We define therapeutic targets as the conditions of illness expressed by the community, which do not always follow the biomedical model of disease. The therapeutic targets may include symptoms, such as inflammation and fever, or a set of symptoms that constitute a more complex condition, such as pneumonia or cancer. Considering that the conditions indicated by the people themselves best describe a local medical system (see Beiersmann et al. 2007; Ferreira Júnior et al. 2011), we suggest that the researcher use "therapeutic targets" as a study unit instead of "illnesses" to facilitate data standardization and comparison of results among different local systems.

Data can be collected through semi-structured interviews, applying the technique of free listing, to obtain from each respondent the number of therapeutic targets treated with plants in addition to the number of plants for each therapeutic target, as a measure of redundancy (see Albuquerque \& Oliveira 2007; Ferreira Júnior et al. 2011). At the conclusion of this stage, one has a general view of the treatment strategies involving medicinal plants that compose the local medical system. From this perspective, one can infer the resilience of the system. A system rich in knowledge of how to treat locally recognized therapeutic targets can be considered only slightly vulnerable to the disturbances noted in the two scenarios described; i.e., the system is resilient. One should remember that the sampling should be representative of the proportion of gender and age classes of individuals, 
which will be of fundamental importance to consider the variations in the distribution of knowledge.

The analysis in a second phase will focus on the individual. The aim of this stage is to determine how much the knowledge of each individual reflects the information compiled in the local system. At this point, we separate the approaches present in the two scenarios only by didactic nature. In the scenario in which the only disturbance assessed is the illness, we consider the following hypothetical case: a system contains 150 therapeutic targets that are treated with plants. One individual recognizes approximately 70 targets that can be treated by using plants, while another individual only reports two targets present in the system. It is accepted that the former respondent is less vulnerable than the latter because he/she holds more knowledge on the use of medicinal plants that can be applied to different targets. To evaluate the proposed hypothesis, the researcher should enter the number of therapeutic targets reported by each respondent into a single column in a database. Using this column, one can perform a normality test to observe the normality of the data in a frequency histogram of respondents based on the number of therapeutic targets reported. Important normality tests that can be applied are the Shapiro-Wilk test, for samples of up to 50 individuals, or the Kolmogorov-Smirnov test, for samples of more than 50 individuals. After applying the test, if the data distribution is normal, the proposed hypothesis is accepted because most of the individuals are distributed around the mean number of reported therapeutic targets treated with medicinal plants (Figure 1A).

For the scenario in which the disturbance assessed can affect the availability of local medicinal species, one should obtain the number of plant species that the individual knows for each therapeutic target that he/she reported. After obtaining that number for each target, the following formula can be used to quantify redundancy:

$$
R i=\frac{\sum e}{N}
$$

Where $R i$ is the measure of the redundancy of therapeutic targets reported by the person $i$, in other words, the sum of the number of species $e$ reported for each target divided by the total number of targets $N$ reported by the respondent. The minimum value obtained using this formula is 1.0 (when the individual reports only one species for each therapeutic target that he/she indicated, showing that there is no redundancy in the categories). An increase of the value of this index represents an increase in the redundancy of the designated medicinal categories.

Below is an example to demonstrate how the calculation is performed: in this case, a respondent indicated three therapeutic targets (fever, migraine and inflammation), with four species for each target; therefore, this respondent would have a value of 4.0 , calculated as follows:

$$
R i=\frac{4+4+4}{3}=4
$$

To test the proposed hypothesis, a column containing the values obtained for $R i$ from each respondent can be constructed and subsequently subjected to a normality test. After applying the test, one can assess whether the distribution of the 
respondents based on the $R i$ values is normal, in which case the proposed hypothesis is accepted because most of the individuals are distributed around the mean for the number of species reported for each therapeutic target. A different situation would be found for a non-normal distribution, which would show a large number of individuals who reported few species for each therapeutic target (low redundancy/more vulnerable individuals) and also a large number of respondents reporting many species (high redundancy/less vulnerable individuals) (Figure 1).

\section{Implications of the hypothesis}

If the proposed hypothesis is true in at least one of the two proposed scenarios, this result suggests a system in which people are adapted to illness events. If the hypothesis is not true, some features of the system can be studied. In this case experts may exist in a community, such as shamans or healers, among others, who may hold the knowledge of plants used for treating illnesses. Additionally, other factors such as gender, age, income can influence the distribution of knowledge of medicinal plants, which may be important generators of discrepancies in the data distribution and should be taken into consideration by the researcher when testing the proposed hypothesis.

One application of our approach is that the present hypothesis can be tested by comparing different medical systems. For example, a comparative analysis of two or more systems can in the future determine which factors (cultural, environmental, social, among others) can explain systems with individuals who reinforce or do not reinforce the resilience of those systems.

For ethnobotanical studies, this approach is a first step to understanding how individuals are resilient in terms of the use of medicinal plants within a local medical system. The present method is not a conclusive approach for assessing resilience at an individual level, given the complexity involved in assessing an individual's resilience (see Ungar 2011, 2012), but it provides an initial proposal using an analytic tool to initiate discussion and can also be applied in areas of medical anthropology, which also seek to study the structure and function of medical systems (Berlin \& Berlin 2005; Waldstein \& Adams 2006).

\section{Acknowledgements}

We thank to CNPq for the financial aid ("Edital Universal" 2012) and productivity grant for research awarded to UPA.

\section{References}

Albuquerque UP, Oliveira RF (2007) Is the use-impact on native caatinga species in Brazil reduced by the high species richness of medicinal plants? Journal of Ethnopharmacology 113:156-170.

Alencar NL, Araújo TAS, Amorim ELC, Albuquerque UP (2010) The inclusion and selection of medicinal plants in traditional pharmacopoeias evidence in support of the diversification hypothesis. Economic Botany 64:68-79. 
Ankli A, Sticher O, Heinrich M (1999) Yucatec Maya medicinal plants versus nonmedicinal plants: indigenous characterization and selection. Human Ecology 27:557-580.

Beiersmann C, Sanou A, Wladarsch E, De Allegri M, Kouyaté B, Müller O (2007) Malaria in rural Burkina Faso: local illness concepts, patterns of traditional treatment and influence on health-seeking behaviour. Malaria Journal 6:106.

Brown PJ (1987) Microparasites and macroparasites. Cultural Anthropology 2:155-171.

Cavalli-Sforza LL, Feldman MW (1981) Cultural transmission and evolution: A quantitative approach. Princeton University Press, Princeton.

Dunn F (1976) Traditional Asian medicine and cosmopolitan medicine as adaptive systems. In: Leslie C (ed) Asian medical systems: a comparative study. University California Press, California, pp: 133-158.

Ferreira Júnior WS, Ladio AH, Albuquerque UP (2011) Resilience and adaptation in the use of medicinal plants with suspected anti-inflammatory activity in the Brazilian Northeast. Journal of Ethnopharmacology 138:238-252.

Hanazaki N, Tamashiro JY, Leitão-Filho HF, Begossi A (2000) Diversity of plant uses in two Caiçara communities from the Atlantic Forest coast, Brazil. Biodiversity and Conservation 9:597-615.

Heinrich J, McElreath R (2003) The evolution of cultural evolution. Evolutionary Anthropology 12:123-135.

Hopkins AL, Stepp JR (2012) Distribution of herbal remedy knowledge in Tabi, Yucatan, Mexico. Economic Botany 66:249-254.

Holling CS (1973) Resilience and stability of ecological systems. Annual Review of Ecology and Systematics 4:1-23.

Kleinman A (1978) Concepts and a model for the comparison of medical systems as cultural systems. Social, Science \& Medicine 12:85-93.

Ladio AH, Lozada, M (2004) Patterns of use and knowledge of wild edible plants in distinct ecological environments: a case study of a Mapuche community from northwestern Patagonia. Biodiversity and Conservation 13:1153-1173.

Ladio AH, Lozada M (2008) Medicinal plant knowledge in rural communites of North-Western Patagonia, Argentina. A resilient practice beyond acculturation. In: Albuquerque UP, Ramos MA (eds) Current topics in Ethnobotany. Research Signpost, Kerala, India, pp. 39-53.

Ladio AH, Lozada M (2009) Human ecology, ethnobotany and traditional practices in rural populations inhabiting the Monte region: Resilience and ecological knowledge. Journal of Arid Environments 73:222-227.

Quinlan MB, Quinlan RJ (2007) Modernization and medicinal plant knowledge in a Caribbean horticultural village. Medical Anthropology Quarterly 21:169-192.

Silva FS, Ramos MA, Hanazaki N, Albuquerque UP (2011) Dynamics of traditional knowledge of medicinal plants in a rural community in the Brazilian semiarid region. Revista Brasileira de Farmacognosia 21:382-391.

Stepp JR, Moerman DE (2001) The importance of weeds in ethnopharmacology. Journal of Ethnopharmacology 75:19-23.

Ungar M (2011) The social ecology of resilience: addressing contextual and cultural ambiguity of a nascent construct. American Journal of Orthopsychiatry 81:1-17.

Ungar M (2012) The social ecology of resilience. A handbook of theory and practice. Springer, New York. 
Ferreira Júnior et al. The role of individuals in the resilience of local medical systems based o the use of medicinal plants - a hypothesis. Ethnobio Conserv 2:1

Waldstein A, Adams C (2006) The interface between medical anthropology and medical ethnobiology. Journal Royal Anthropological Institute N.S.:S95-S118.

Wiley SA (1992) Adaptation and the biocultural paradigm in Medical Anthropology: a critical review. Medical Anthropology Quarterly 6:216-236. 